\title{
Software for Static Equipment Budgeting of China's Petrochemical Industry
}

\author{
Rong L* \\ SINOPEC Engineering Inc, China
}

*Corresponding author: Li Rong, Senior Engineer, Cost Estimate Department in SINOPEC Engineering Inc, China, Tel: +86-10-84875185; Email: lirong@sei.com.cn

\section{Research Article}

Volume 5 Issue 4

Received Date: August 27, 2021

Published Date: October 13, 2021

DOI: $10.23880 /$ ppej-16000279

\section{Abstract}

We developed software by using Python language to reduce the manual work in the petrochemical budgeting process. Budget forms automatically generated by the software are based on the acquisition cost of items of static equipment and their installation fees. The software is also able to generate an Excel file that can be imported into the petrochemical aggregation software. This article explains the logic of the software based on the static equipment workflow in the current project. It provides development ideas to solve the problem of manually inputting equipment installation indexes, avoid confusion of equipment prices in the traditional estimate preparation process, and improve the work efficiency in the future.

Keywords: Budget Estimation; Python; Automatic Budgeting Process; Petrochemical Project Cost

\section{Introduction}

From the perspective of investment, the management of petrochemical engineering mainly includes the following stages: the investment estimation in the pre-project stage, the estimate in the construction drawing stage, the project settlement, and final account after completion. The price of equipment accounts for as much as $60 \%-70 \%$ of total cost, which an important factor affecting the cost of petrochemical is engineering. The professional cost of equipment is usually composed of equipment acquisition cost and installation cost. Among them, the equipment acquisition cost is mainly calculated according to the Equipment Price Information [1] during the design period, and the installation cost is calculated by the estimated installation of petrochemical project.

Many researchers have done a lot of works in the field of estimation. Cui [2] analyzed the problems existing in the preparation of petrochemical project budget estimate, finds out the influencing factors of terminal budget estimate investment. Wan [3] analysed the cost control process of EPC boiler project of petrochemical system with the practice of boiler project of an ethylene project of Sinopec.

There are many factors affecting the cost of the estimates. It often requires significant manual work to process all of them. However, the logic of these factors are easy to follow and well documented. By studying the index system of petrochemical engineering, the components of installation costing could be clarified. The solution to improve the efficiency of equipment estimate preparation is to rely on computer software for automatic batch processing. It can save more than $80 \%$ of the labor hours invested in the estimation of static equipment.

Python [4] is a cross-platform computer software programming language. It is a high-level combination of interpreted, compiled, interactive, and object-oriented scripting language. Although originally designed for writing 


\section{Petroleum \& Petrochemical Engineering Journal}

automated scripts, it is now widely used for independent, large-scale petrochemical project development as more and more new features are added to the language.

In this paper, we will use previous engineering projects to explain in detail the idea and logic of the software development, taking the column in the static equipment as an example. The total cost of static equipment could be divided into two parts: one is the installation fees which could be calculated by static equipment budget index, and the other one is the cost of equipment acquisition which could be calculated by the unit price.

\section{Software Logic for Index Application of Static Equipment Installation}

In the project application database, the installation of static equipment is classified in section 1: column and vessel. This includes the installation of common static equipment such as columns, column trays, reactors, heat exchangers, air coolers, vessels. A schematic figure of shell material is shown in Figure 1. The process logic will be described using the column as an example.

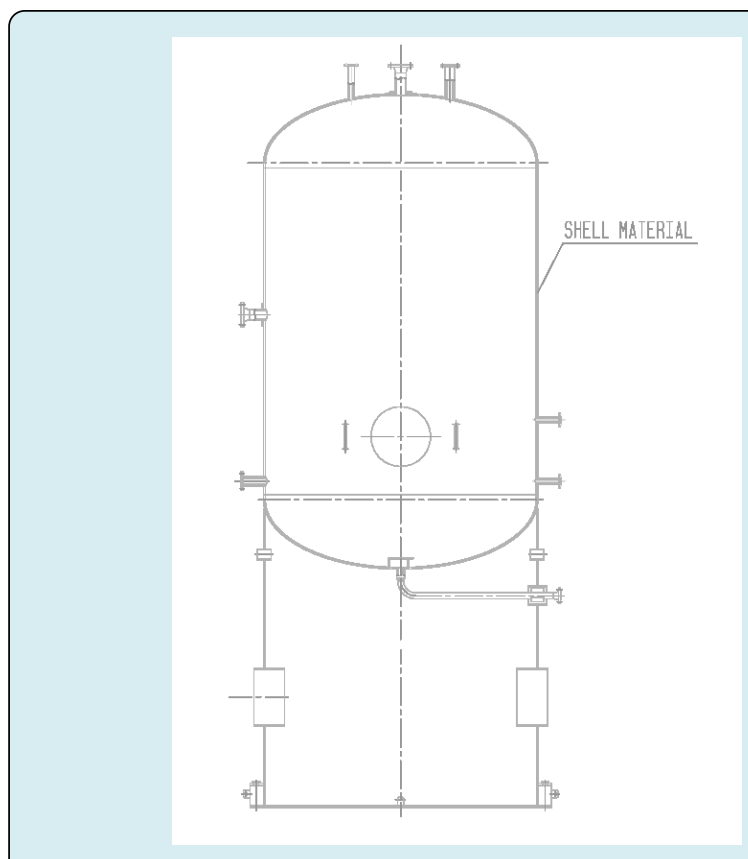

Figure 1: Schematic figure of shell material.

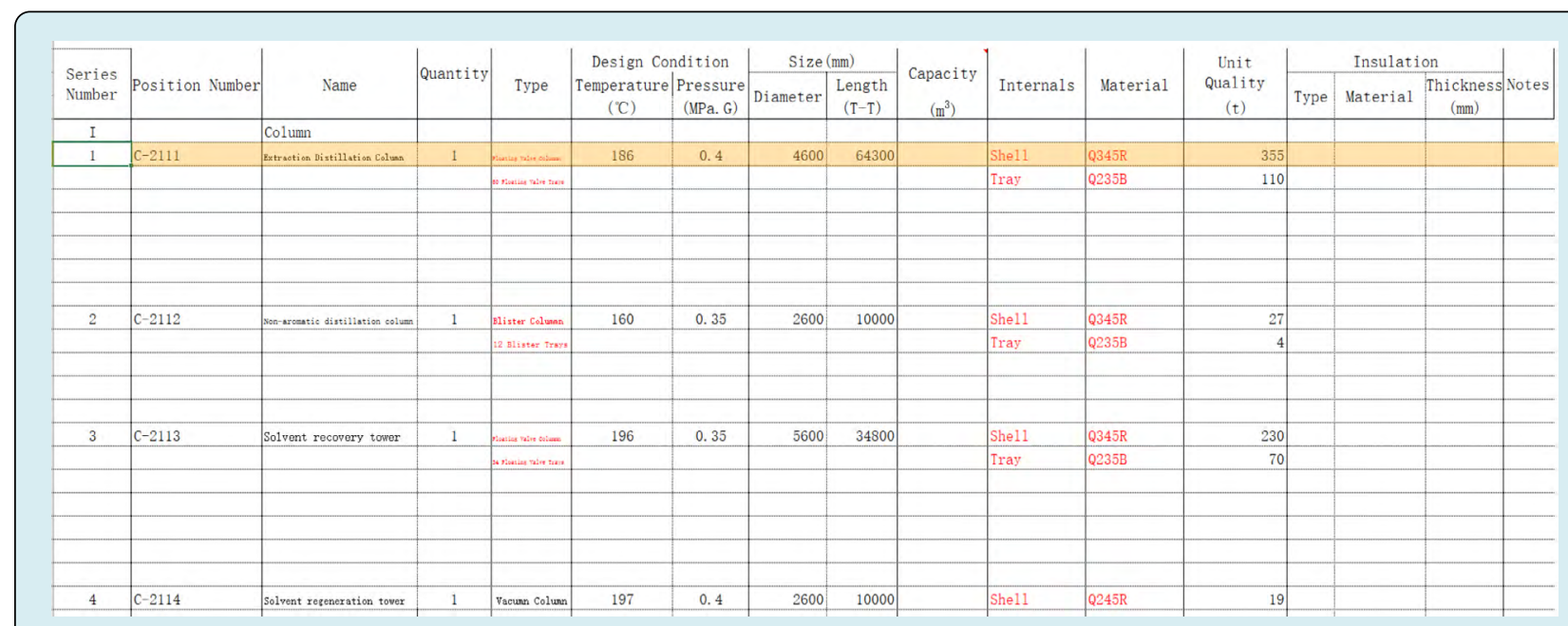

Figure 2: Process Equipment Datasheet.

Figure 2 shows the datasheet received from the upstream majors. The columns are usually divided into two parts, the shells and the internals. The datasheet also provides conditions such as the type and size of the equipment.

\section{Installation of the Shell}

Classification of shell material selection: The material of the column shell in the software is classified. The materials are divided into carbon steel, alloy steel, stainless steel and other materials. And the common material numbers are included in the database of shell materials. Table 1 shows some of these materials and their designations. The software will apply the corresponding index according to the "material" in the row. For carbon steel columns, the base price is applied. Aluminium columns are adjusted by multiplying the overall index price by a factor of 1.3 . 


\section{Petroleum \& Petrochemical Engineering Journal}

\begin{tabular}{|c|c|c|c|c|c|}
\hline $\begin{array}{c}\text { Serial } \\
\text { number }\end{array}$ & Material & Code 1 & Code 2 & Code 3 & Code 4 \\
\hline (1) & Carbon steel Q245R & & Q245R & $20 \mathrm{R}$ & $20 \mathrm{~g}$ \\
\hline$(2)$ & Low alloy steel Q345R & & Q345R & $16 \mathrm{MnR}$ & $16 \mathrm{Mng}$ \\
\hline (4) & $\begin{array}{c}\text { Stainless steel 0Cr18Ni9 } \\
(304)\end{array}$ & S30408 & 06Cr19Ni10 & $0 \mathrm{Cr} 18 \mathrm{Ni} 9$ & 304 \\
\hline (5) & $\begin{array}{c}\text { Stainless steel } \\
\text { 0Cr18Ni10Ti (321) }\end{array}$ & S32168 & 06Cr18Ni11Ti & 0Cr18Ni10Ti & 321 \\
\hline (6) & $\begin{array}{c}\text { Stainless steel } \\
\text { 00Cr17Ni14Mo2 (316L) }\end{array}$ & S31603 & 022Cr17Ni12Mo2 & 00Cr17Ni14Mo2 & $316 \mathrm{~L}$ \\
\hline (7) & $\begin{array}{l}\text { Aluminium L1 to L6, LF1 } \\
\text { to LF2 }\end{array}$ & & & L1 to L6 & LF1 to LF2 \\
\hline (9) & $\begin{array}{c}\text { Clad sheet steel (carbon } \\
\text { steel }+304 \text { ) }\end{array}$ & $\mathrm{Q} 245 \mathrm{R}+30408$ & $Q 345 R+30408$ & Q245R+06Cr19Ni10 & Q345R+06Cr19Ni10 \\
\hline$(10)$ & $\begin{array}{c}\text { Clad sheet steel (carbon } \\
\text { steel + 316L) }\end{array}$ & $\mathrm{Q} 245 \mathrm{R}+32168$ & $Q 345 R+32168$ & $\begin{array}{c}\mathrm{Q} 245 \mathrm{R} \\
+022 \mathrm{Cr} 17 \mathrm{Ni} 12 \mathrm{Mo} 2\end{array}$ & $\begin{array}{c}\mathrm{Q} 345 \mathrm{R} \\
+022 \mathrm{Cr} 17 \mathrm{Ni} 12 \mathrm{Mo} 2\end{array}$ \\
\hline
\end{tabular}

Table 1: Designations corresponding to selected column shell materials.

Software logic of the shell installation: There are three methods for column installation: piecewise, segmental and integral installations. The specific installation methods are chosen according to Table 2 shown below. The procedure can also be modified according to the actual adopted standard.

\begin{tabular}{|c|c|c|}
\hline & $\begin{array}{c}\text { Diameter greater than } \\
\text { or equal to } 3.8 \mathrm{~m}\end{array}$ & $\begin{array}{c}\text { Diameter less } \\
\text { than } 3.8 \mathrm{~m}\end{array}$ \\
\hline $\begin{array}{c}\text { Height less } \\
\text { than } 20 \mathrm{~m}\end{array}$ & Installation in pieces & $\begin{array}{c}\text { Overall } \\
\text { installation }\end{array}$ \\
\hline $\begin{array}{c}\text { Height greater } \\
\text { than } 20 \mathrm{~m}\end{array}$ & Installation in pieces & $\begin{array}{c}\text { Segmented } \\
\text { installation }\end{array}$ \\
\hline
\end{tabular}

Table 2: Column field installation methods.

Software logic for shell field assembly welding: For the installation of the column shell in pieces and segments, the calculation of local heat treatment work after welding is required. The number of segments and pieces of the column body needs to be provided by the upstream specialist. If the upstream specialist does not provide these information, the software defaults to dividing the shell into 2 pieces and 2 sections, and calculates the length of the required heat treatment welds by the shell diameter and height.

It should be noted that the weld involved is the length of the weld that needs to be heat treated at the construction site. It is not the length of the weld that is heat treated during fabrication of the column in the fabrication shop. The cost of heat treatment during the manufacturing process is included in the price of the equipment material.

\section{Installation of the Column Tray}

The installation of the column tray is classified according to its type as floating valve tray, blister tray, tongue tray, hybrid tray, etc. After reading the column type from the equipment table, the column index is applied according to the column body diameter. For the column type not given in the equipment datasheet, the default type is floating valve column.

\section{Static Equipment Price Inquiry}

The Price Information of Equipment is given in the Engineering and Economic Information published by the Technical Central Station of the China Petroleum and Chemical Corporation for Budget Estimation. The prices of each period are entered into the database of the software. Based on the fact that the price information is updated with time, the software provides a date option, thus facilitating the designer to select the appropriate price. It also facilitates the maintenance and updating of the price database by the budget estimator. As long as the latest issue of price information is placed in the database, the selection of prices for different periods can be made. The overall estimate prices generated can also be easily compared with those of previous periods.

\section{Logic for Equipment Price Queries}

We need to determine the category of the device before matching the price. The software has a database embedded in it to determine the category of the container based on the information given in the equipment table. 


\section{Petroleum \& Petrochemical Engineering Journal}

The equipment category is determined by the medium classification inside the equipment, the design pressure and the volume. The software reads the working medium, design pressure, internal diameter and height from the equipment table and determines the equipment category through the embedded formulas and database.

Once the column category is determined, you can find the material prices for columns in the Equipment Price
Information. All columns are priced as empty columns.

\section{Logic for Trays Price Query}

The price of the column trays is calculated by referring to the price of the internal parts in the Equipment Price Information. For different material grades, a database has also been created in the software for classification and mapping (Table 3).

\begin{tabular}{|c|c|c|c|c|c|}
\hline Serial number & Materials & Code 1 & Code 2 & Code 3 & Code 4 \\
\hline$(1)$ & Q245 Inner Parts & & Q245R & 20R & $20 \mathrm{~g}$ \\
\hline$(2)$ & Q345 Inner Parts & & Q345R & 16MnR & 16Mng \\
\hline$(3)$ & 304 Inner Parts & S30408 & 06Cr19Ni10 & 0Cr18Ni9 & 304 \\
\hline$(4)$ & 316 Inner Parts & S32168 & 06Cr18Ni11Ti & 0Cr18Ni10Ti & 321 \\
\hline
\end{tabular}

Table 3: Designations for selected column internals materials.

\section{Software Programming for Automated Batch Processing}

\section{Using hash tables to store structured bulk information}

In this application scenario, various datasheets contain various structured batch information. For example, one datasheet contains structural relationship between code, issue number and price in the database, and another datasheet contains structural relationship between budge index, static equipment and specification.

When dealing with equipment index application and price queries, it is necessary to frequently look up a specific index from these datasheets. During the software development, we take full advantage of the large memory capacity of computers nowadays. By using hash tables to store this structured bulk information, which reduces the complexity of the lookup process from linear level to constant level, it greatly reduces the performance overhead in the lookup and improving the execution speed of the software.

\section{Automatic Identification of Equipment}

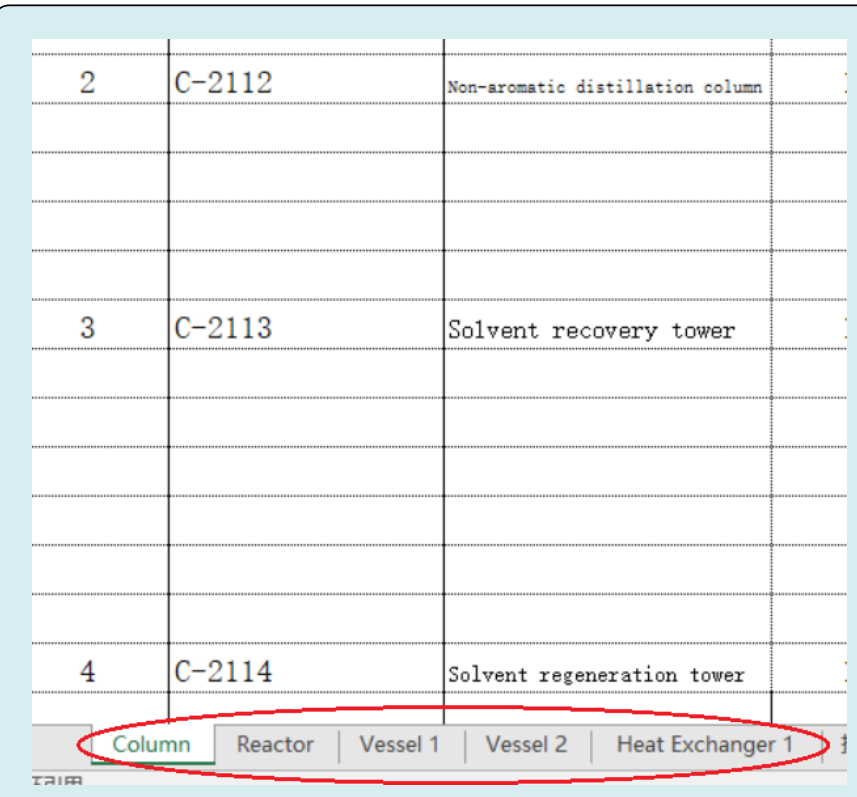

Figure 3: Contents of the column in the equipment table. 


\section{Petroleum \& Petrochemical Engineering Journal}

The software automatically identifies and extracts the equipment type and the specific items of equipment contained in the equipment type from the equipment datasheet. Taking Figure 3 as an example, the software can automatically identify four major types of equipment: columns, reactors, vessels and heat exchangers. In addition, in the "Column" table, the software can also automatically identify: extraction distillation column (106-C-2111), nonaromatic distillation column (106-C-2112) and so on. The whole process does not require user participation.

\section{Issue the Price of Automatic Extraction Equipment}

The software automatically identifies and extracts the price period numbers of various equipment types from the “Equipment Price Database", e.g., 200701, 201903, as shown in the Figure 4.

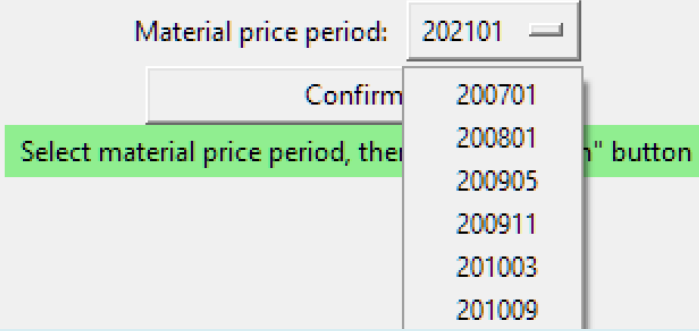

Figure 4: Price Inquiry Screen.

Since the extraction of price period numbers is completely automatic, even if new price period numbers are added to the "Equipment Price Database" in the future, the software will automatically identify and display the new price period numbers without any manual work. The results of the program application on devices are shown in Figure 5.

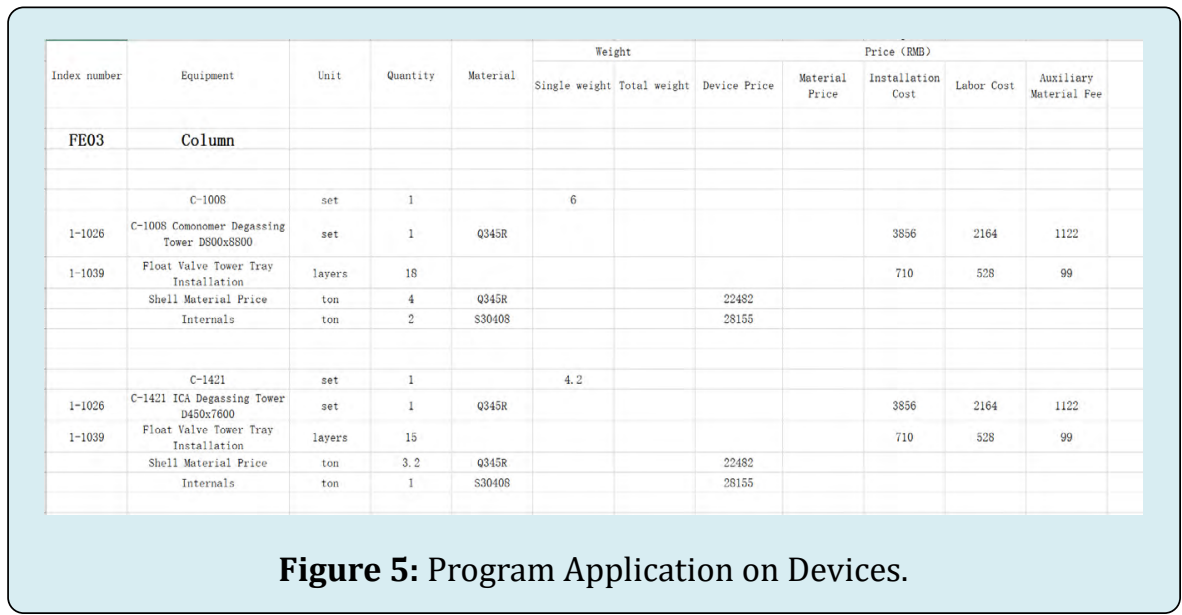

\section{Automatic Invocation of the Corresponding Index Application Logic}

For the purpose of reusability and maintainability, the software is coded in such a way that the index application logic and price search logic are abstractly encapsulated. The software will automatically call the corresponding index application logic and price search logic according to the device name.

For example, if the equipment is a column, it will automatically call the column index number query function, which will automatically extract the weight, diameter and height of the column equipment from the datasheet, and determine the installation method of the equipment from these index (piecewise group installation, sectional group installation, or overall installation). The index number of the corresponding weight will also be adopted.

If the equipment is a reactor, it will automatically call the reactor type index number query function, automatically extract the design pressure and weight of the reactor type equipment from the table, use these indexes to determine the installation method of the equipment (low and medium pressure reactor installation, or high pressure reactor installation), and the corresponding weight specifications of the index number. Similar logic will be followed for other equipment.

By encapsulating the index application logic and price search logic at the code level, it reduces the cost of understanding the index source code for developers and principals, and facilitates future updates and maintenance of the index.

\section{Summary}

This paper focuses on the working principle and the logic of software. Using this software in the work, it can significantly save the working time and improve the efficiency. The compiled files can also be imported into the project database in the subsequent work, laying a good 


\section{Petroleum \& Petrochemical Engineering Journal}

foundation for future analysis and forecasting of the total project budget. It allows for a more comprehensive range of estimates and greater accuracy.

\section{References}

1. Nonstandard Equipment Price Information (2020) Engineering Economic Information 1(1): 33-35.

2. Caixia C (2021) Budget Estimate Compilation and
Analysis of LNG Terminal Project. ShanDong Chemical Industry 50: 154-157.

3. Xiong W (2016) Analysis of Measures to Improve the Economic Efficiency of Boiler EPC Projects in Petrochemical Systems. China Petrochem, pp: 106-108.

4. Python. 